\title{
Local recurrent vaginal aggressive angiomyxoma misdiagnosed as cellular angiomyofibroblastoma: A case report
}

\author{
YIN-FENG WANG, HONG-LANG QIAN and HANG-MEI JIN \\ Department of Gynecology, Women's Hospital, School of Medicine Zhejiang University, \\ Hangzhou, Zhejiang 310006, P.R. China
}

Received December 29, 2014; Accepted January 11, 2016

DOI: 10.3892/etm.2016.3097

\begin{abstract}
Aggressive angiomyxoma (AAM) and angiomyofibroblastoma (AMFB) are two rare types of mesenchymal tumors with overlapping clinicopathological features. In certain cases, the differential diagnosis between the two tumors is difficult even for experienced pathologists. The present study reported the case of a well-circumscribed soft tissue mass on the anterior wall of the vagina in a 25 -year-old woman. The mass was initially removed without disturbance to the adjacent tissues. The histopathological features included spindle cells in inconspicuous myxoid stroma and a well-demarcated mass without evidence of invasion, which prompted the initial diagnosis of AMFB. After 2 years, a mass returned in the same area and a wide tumor excision was performed. The histopathological examination confirmed the final diagnosis of AAM upon review.
\end{abstract}

\section{Introduction}

Aggressive angiomyxoma (AAM) and angiomyofibroblastoma (AMFB) are rare types of mesenchymal neoplasms, with a marked predilection for the female genital tract, in particular, the vulva and vagina. There has been a limited number of reports concerning AAM or AMFB individually since AAM was initially described by Steeper and Rosai in 1983 (1-5). The prevalence rate of AAM and AMFB is unclear due to the rarity of the condition. AMFB may be treated by surgical removal of the tumor without reported recurrence $(6,7)$. While AAM is a slow-growing, but locally invasive, uncapsulated neoplasm, relapse may still occur following wide excision of the tumor $(4,8)$. AMFB may be treated by surgical removal of the tumor, while relapse may occur in patients with AAM even after a wider excision of the tumor (4). Hence, differential

Correspondence to: Professor Hang-Mei Jin, Department of Gynecology, Women's Hospital, School of Medicine Zhejiang University, 1 Xueshi Road, Hangzhou, Zhejiang 310006, P.R. China E-mail: jinhm@zju.edu.cn

Key words: misdiagnosis, recurrence, aggressive angiomyxoma, cellular angiomyofibroblastoma diagnosis between the two entities is crucial for surgical planning. In certain rare cases, this differential diagnosis is difficult even for experienced pathologists due to the overlapping morphological features. The present study reported a case of well-circumscribed AAM misdiagnosed as cellular AFMB, which was treated with a simple resection and the patient presented a local relapse after 2 years.

\section{Case report}

A 25-year-old woman was initially admitted to the Department of Gynecology, Women's Hospital, School of Medicine Zhejiang University (Hangzhou, China) in April 2010, and again in March 2012. The patient presented with a painless swelling in the anterior wall of the vagina, incidentally detected during a routine gynecological examination. A palpable, non-tender, solid mass was identified on the anterior wall of the vagina upon the patient's initial admission. The mass was freely movable in relation to the adjacent tissues. Ultrasound examination (Voluson 730 Ultrasound System; GE Healthcare Life Sciences, Chalfont, UK) revealed that the mass was a well-circumscribed soft tissue mass with heterogeneous internal echoes. The mass was measured to be $\sim 3.3 \times 3.7 \times 3.4 \mathrm{~cm}$ in size and protruded toward the bladder, with rich intralesional vascularization observed by color Doppler (Fig. 1). The tumor was removed in full without damage to the adjacent structures; the tumor was diagnosed as leiomyoma. Upon sectioning, the tumor was well-demarcated with a gelatinous appearance, with no foci of hemorrhage or necrosis. The patient received no further treatment as the pathological diagnosis at the time was AMFB.

After 2 years, the patient was admitted to the Women's Hospital, School of Medicine Zhejiang University (Hangzhou, China), with a chief complaint of frequent micturition. A gynecological examination revealed a diffused mass in the anterior wall of the vagina. The mass was rubbery to firm in consistency and mildly tender on palpation. Ultrasonography indicated that the hypoechoic mass, $4.9 \times 3.6 \times 3.0 \mathrm{~cm}$ in size, was circumscribed but not well-demarcated between the paries anterior vagina and posterior bladder wall. Color Doppler flow imaging revealed that the arterial blood flow was of predominantly low resistance (Fig. 2). The patient underwent a wide tumor excision and repair of the vesicovaginal fistula, as part of the adhesive posterior bladder wall was incised during 


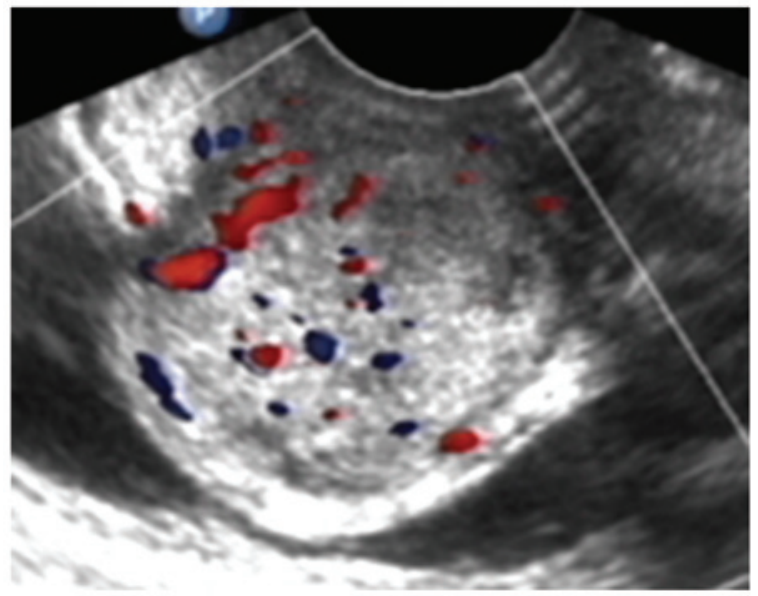

Figure 1. Transvaginal ultrasound revealed a well-circumscribed soft tissue mass with heterogeneous internal echos. The size of the mass was $3.3 \times 3.7 \times 3.4 \mathrm{~cm}$, with rich intralesional vascularization. The blood flow was investigated using color Doppler.

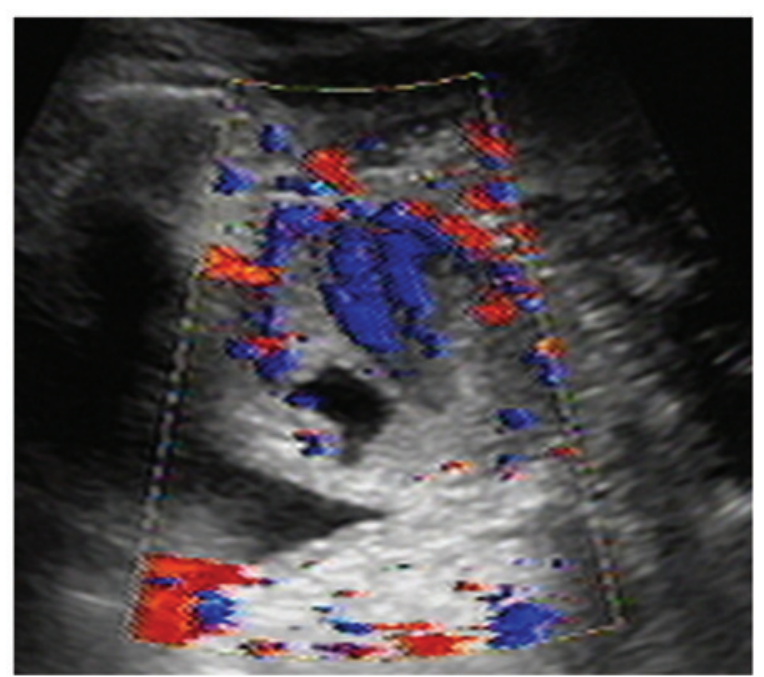

Figure 2. Transvaginal ultrasound demonstrated a hypoechoic circumscribed but not well-demarcated mass. Color Doppler flow imaging revealed predominantly low resistance type arterial blood flow between the paries anterior vagina and posterior bladder wall. The size of the mass was $4.9 \times 3.6 \times 3.0 \mathrm{~cm}$.

the surgery. Upon gross examination, the excised specimen was irregular and partially capsulated. The tumor appeared to focally extend into the surrounding normal tissues and displayed a rubbery, glistening grey-white surface. The final pathological diagnosis was AAM. The patient remained free from tumor throughout the 3-year follow-up period; the final follow-up was in May 2015.

The specimens from both surgeries were spliced grossly into samples $2 \times 2 \times 0.2 \mathrm{~cm}$, cut $(4 \mu \mathrm{m})$ using a rotary microtome (Micron HMB40E; Thermo Fisher Scientific, Inc., Waltham, MA, USA), and stained with hematoxylin and eosin (Shenzhen Boao Biotechnology Co., Ltd., Shenzen, China). The slides were observed under a microscope (DMR 2000; Leica Microsystems, Wetzlar, Germany). The typical histopathological changes in the initial and second surgeries are displayed in Fig. 3, respectively. Common morphological features including cellular spindle cells and abundant small
A

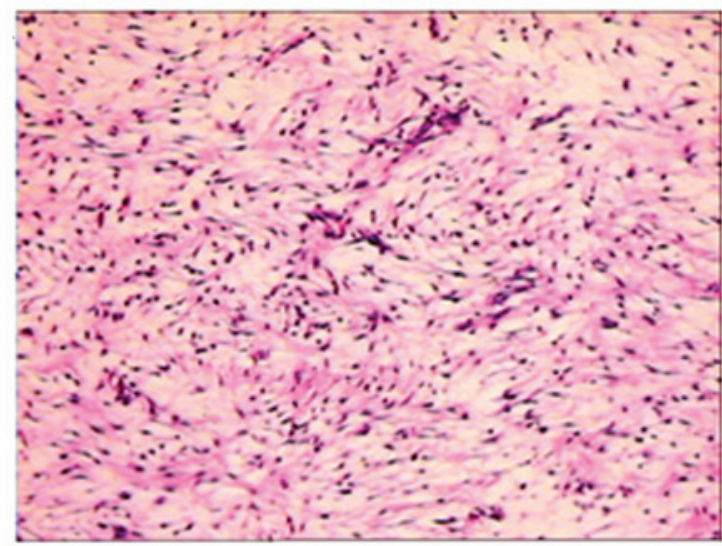

$\mathbf{B}$

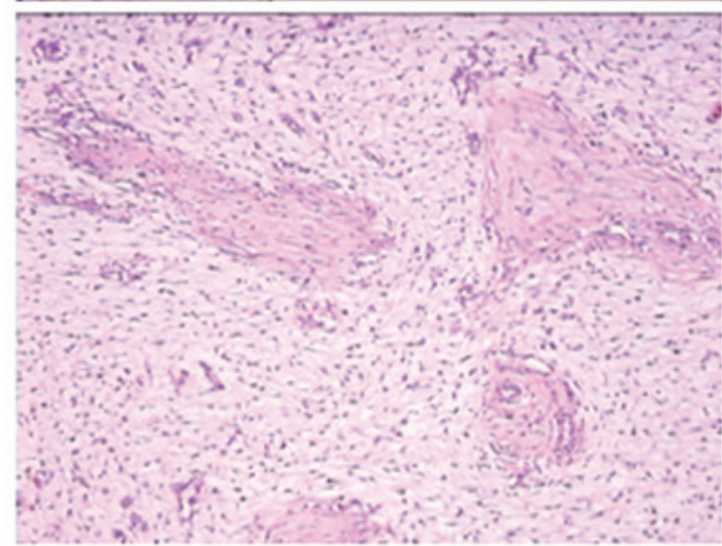

Figure 3. Histopathological changes indicated similar morphological features in the (A) initial and (B) second surgeries, including cellular spindle cells and small blood vessels in the myxoid stroma. However, the specimen from the second surgery displayed sparser cells and more prominent myxoid stroma, compared with the initial surgery specimen. The small elastic vessels in the two figures is not a typical feature of AAM; they are more frequently observed in AMFB. Magnification, x100.

blood vessels in the slightly myxoid milieu were evident. The well-demarcated mass, which did not show evidence of involving the surrounding normal tissue and inconspicuous myxoid stroma, prompted the initial diagnosis of AMFB, although the classical concentrated pattern around the vessel was not prominent. The recurrence of the tumor, invasion into the surrounding tissue and the evident myxoid components in the second surgery provided substantial evidence for the diagnosis of AAM by the pathologists.

\section{Discussion}

AMFB is a relatively rare soft tissue tumor characterized by benign biological behavior. Previously published studies concerning patients with AMFB have presented benign tumors without local recurrence or metastatic potential, even after a prolonged follow-up period of 5 years (6-8). In contrast to AMFB, AAM is a more infiltrative neoplasm and has a high propensity for local recurrence. The local recurrence of AAM varies in the range of $36-72 \%$ according to the extent of resection during the initial surgery or the location of the tumor $(9,10)$.

Differential diagnosis for the two lesions is important for the subsequent treatment, as the prognoses are different. AAM and AMFB are associated neoplasms in a spectrum of 
tumors displaying a myofibroblastic origin (11). Furthermore, immunohistochemical examination is of limited value in discriminating between the two tumors, while histomorphological analysis is the principal method of distinction $(1,12,13)$. The histopathological appearance of AMFB includes a prominent thin-walled vessel and round-to-spindle cells in a background of abundant loose edematous stroma. The tumor cells are typically concentrated to the areas surrounding the vessels. By contrast, AAM is composed of stellate, spindle cells in a loosely collagenous myxoedematous matrix with scattered vessels. However, the two lesions also display overlapping histopathological features when the vessels are less prominent in AMFB. Under such circumstances, gross examination is the key to differential diagnosis. AMFB is well-circumscribed, while AAM frequently adheres to fatty, muscle and regional structures.

In the present case, the pathological morphology was not typical of AAM in the initial surgical specimen. Histopathological features including cellular spindle cells and small blood vessels in the somewhat myxoid matrix did not provide sufficient evidence to identify AAM or AMFB. The boarders in the resected tumor was unable to adequately indicate the invasion characteristics under the microscope due to the absence of surrounding tissue. AMFB is much more cellular, and has a less conspicuous myxoid stroma, than typical AAM. The aforementioned peculiarities observed in the present case were similar to those observed in AMFB, which lead to the misdiagnosis. However, the biological behavior of local recurrence and histopathological features of relatively larger, thin-wall blood vessels resulted in the final diagnosis of AAM.

In conclusion, AAM and AMFB are rare mesenchymal neoplasms with overlapping features. Histological delimitation is the predominant marker for differential diagnosis, which is difficult when the vessels are less prominent in AMFB. In the present study, gross examination with local invasion served a significant role in the diagnosis of AAM. Therefore, the present study suggests that wide excision is necessary for decreasing local recurrence and providing the pathologist with sufficient information to achieve a correct diagnosis of AAM or AMFB.

\section{References}

1. Steeper TA and Rosai J: Aggressive angiomyxoma of the female pelvis and perineum. Report of nine cases of a distinctive type of gynecologic soft-tissue neoplasm. Am J Surg Pathol 7: 463-475, 1983.

2. Bakhtiar UJ and Awan AS: Aggressive angiomyxoma of vulva. J Coll Physicians Surg Pak 23: 507-508, 2013.

3. Ki EY, Park JS, Lee A and Hur SY: Aggressive angiomy xoma of the female genital tract: Report of two cases. Eur J Gynaecol Oncol 35: 465-468, 2014.

4. Sengupta SK, Bhattacharyya SK, Saha SP, Roy H and Sarkar AN: Recurrent aggressive angiomyxoma of the vulva-a rare presentation. J Clin Diagn Res 8: OD01-OD02, 2014.

5. Ducarme G, Valentin M, Davitian C, Felce-Dachez M and Luton D: Angiomyofibroblastoma: A rare vulvar tumor. Arch Gynecol Obstet 281: 161-162, 2010.

6. Tzanakis NE, Giannopoulos GA, Efstathiou SP, Rallis GE and Nikiteas NI: Angiomyofibroblastoma of the spermatic cord: A case report. J Med Case Rep 4: 79, 2010.

7. Kairi-Vassilatou E, Dastamani C, Vouza E, Mavrigiannaki P, Hasiakos D and Kondi-Pafiti A: Angiomyofibroblastoma of the vulva: A clinicopathological and immunohistochemical analysis of a rare benign mesenchymal tumor. Eur J Gynaecol Oncol 32: 353-355, 2011.

8. Kanda M, Sonoyama A, Hirano H, Kizaki T and Ohara N: Angiomyofibroblastoma of the vulva. Eur J Gynaecol Oncol 35: 77-80 2014

9. Fetsch JF, Laskin WB, Lefkowitz M, Kindblom LG and Meis-Kindblom JM: Aggressive angiomy xoma: A clinicopathologic study of 29 female patients. Cancer 78: 79-90, 1996.

10. Begin LR, Clement PB, Kirk ME, Jothy S, McCaughey WT and Ferenczy A: Aggressive angiomyxoma of pelvic soft parts: A clinicopathologic study of nine cases. Hum Pathol 16: 621-628, 1985.

11. Granter SR, Nucci MR and Fletcher CD: Aggressive angiomyxoma: Reappraisal of its relationship to angiomyofibroblastoma in a series of 16 cases. Histopathology 30: 3-10, 1997.

12. Fletcher CD, Tsang WY, Fisher C, Lee KC and Chan JK: Angiomyofibroblastoma of the vulva. A benign neoplasm distinct from aggressive angiomyxoma. The Am J Surg Pathol 16: 373-382, 1992.

13. Mathlouthi N, Slimani O, Soumaya R, Ben Jilani Sarra B, Ben Temime R, Makhlouf T, Abdelhamid K, Attia L and Chachia A: Aggressive angiomyxoma of the female pelvic and perineum. Tunis Med 91: 76-77, 2013 (In French). 